\title{
Analysis of the Eclipsing Binaries in the LMC Discovered by OGLE: Period Distribution and Frequency of the Short-Period Binaries
}

\author{
Tsevi Mazeh ${ }^{1}$, Omer Tamuz ${ }^{1}$ and Pierre North ${ }^{2}$ \\ ${ }^{1}$ School of Physics and Astronomy, Raymond and Beverly Sackler Faculty of Exact Sciences, \\ Tel Aviv University, Tel Aviv, Israel \\ email: mazeh@wise.tau.ac.il \\ ${ }^{2}$ Laboratoire d'Astrophysique, Ecole Polytechnique Fédérale de Lausanne (EPFL), \\ Observatoire, CH-1290 Sauverny, Switzerland
}

\begin{abstract}
.
We review the results of our analysis of the OGLE LMC eclipsing binaries (Mazeh, Tamuz \& North 2006), using EBAS - Eclipsing Binary Automated Solver, an automated algorithm to fit lightcurves of eclipsing binaries (Tamuz, Mazeh \& North 2006). After being corrected for observational selection effects, the set of detected eclipsing binaries yielded the period distribution and the frequency of all LMC short-period binaries, and not just the eclipsing systems. Somewhat surprisingly, the period distribution is consistent with a flat distribution in $\log P$ between 2 and 10 days. The total number of binaries with periods shorter than 10 days in the LMC was estimated to be about 5000. This figure led us to suggest that $(0.7 \pm 0.4) \%$ of the main-sequence A- and B-type stars are found in binaries with periods shorter than 10 days. This frequency is substantially smaller than the fraction of binaries found by smaller radial-velocity surveys of Galactic B stars.
\end{abstract}

Keywords. methods: data analysis, binaries: eclipsing, Magellanic Clouds

\section{Introduction}

The OGLE project yielded a huge photometric dataset of the LMC (Udalski et al. 2000), which includes a few thousand eclipsing binary lightcurves (Wyrzykowski et al. 2003). This dataset allows for the first time a statistical analysis of the population of short-period binaries of an entire galaxy. To analyse this set of lightcurves we constructed EBAS (Eclipsing Binary Automated Solver), an automated algorithm (Tamuz, Mazeh \& North 2006) to fit eclipsing lightcurves. Having solved the lightcurves with EBAS, we proceeded to derive the statistical features of the eclipsing binaries of the LMC (Mazeh, Tamuz \& North 2006). In this short paper we present the EBAS algorithm and show two results of interest: a flat log-period distribution and a lower-than-expected binary frequency of the short-period early-type binaries.

\section{The EBAS Algorithm}

EBAS is based on the EBOP code (Popper \& Etzel 1981, Etzel 1981), which consists of two main components. The first component generates a lightcurve for a given set of orbital elements and stellar parameters, while the second finds the parameters that best fit the observational data. We only used the lightcurve generator, and wrote our own code to search for the best-fit set of elements that minimize the $\chi^{2}$ statistic. The search 
for the global $\chi^{2}$ minimum is performed in two stages. We first find a good initial guess, and then use a simulated annealing algorithm to find the global minimum. While the first stage is merely aimed at finding an initial guess for the next stage, in most cases it already converges to a very good solution. A full description of the algorithm is given in Tamuz, Mazeh \& North (2006).

During the development of EBAS we found that some solutions with low $\chi^{2}$ might be unsatisfactory. While, for such solutions, the value of $\chi^{2}$ is reasonable, a visual inspection of the residuals, plotted as a function of phase, revealed that the fit is sub-optimal. For such cases, human interaction was needed to improve the fit, or to otherwise decree the solution unsatisfactory. In order to allow an automated approach, an automatic algorithm must replace human evaluation.

We therefore defined a new estimator which is sensitive to the correlation between adjacent residuals of the measurements relative to the model. This feature is in contrast to the behaviour of the $\chi^{2}$ function, which measures the sum of the squares of the residuals, but is not sensitive to the signs of the different residuals and their order.

Denoting by $k_{i}$ the number of residuals in the $i$-th run (a series of consecutive residuals with the same sign), we defined the 'alarm' $\mathcal{A}$ as:

$$
\mathcal{A}=\frac{1}{\chi^{2}} \sum_{i=1}^{M}\left(\frac{r_{i, 1}}{\sigma_{i, 1}}+\frac{r_{i, 2}}{\sigma_{i, 2}}+\cdots+\frac{r_{i, k_{i}}}{\sigma_{i, k_{i}}}\right)^{2}-\left(1+\frac{4}{\pi}\right),
$$

where $r_{i, j}$ is the residual of the $j$-th measurement of the $i$-th run and $\sigma_{i, j}$ is its uncertainty. The sum is over all the measurements in a run and then over the $M$ runs. Dividing by $\chi^{2}$ assures that, in contrast to $\chi^{2}$ itself, $\mathcal{A}$ is not sensitive to a systematic overestimation or underestimation of the uncertainties. It is easy to see that $\mathcal{A}$ is minimal when the residuals alternate between positive and negative values, and that long runs with large residuals increase its value.

When a solution found by EBAS showed high $\mathcal{A}$, EBAS automatically classified the solution as unsatisfactory and started a modified search of the parameter space in order to find a better solution. Systems for which a low enough $\mathcal{A}$ solution could not be found were marked as such. Visual inspection of these lightcurves and their fitted models showed that most of them are close contact systems which EBOP did not model well.

\section{Analysis of the OGLE LMC Eclipsing Binaries}

The LMC OGLE-II photometric campaign (Udalski et al. 2000) was carried out from 1997 to 2000, during which between 260 and 512 measurements in the $I$-band were taken for 21 fields (Zebrun et al. 2001). Wyrzykowski et al. (2003) searched the photometric data base and identified 2580 binaries. We analysed those systems with EBAS and found 1931 acceptable solutions. We excluded all binaries with sum of radii larger than 0.6 of the binary separation. Furthermore, we excluded also binaries with high $\mathcal{A}$ values, which indicated that they are probably contact systems that EBOP can not model properly (see Mazeh, Tamuz \& North (2006) for details).

The very large sample of 1931 short-period binaries enables us to derive some statistical features of the population of short-period binaries in the LMC. However, the sample suffers from serious observational selection effects, which affected the discovery of the eclipsing binaries. To be able to correct for the selection effects we needed a well-defined homogeneous sample. We therefore trimmed the sample before deriving the period distribution. Our trimmed sample consisted of all systems of magnitude between 17 and 19 in the $I$ band with periods shorter than 10 days and having main-sequence color (see 


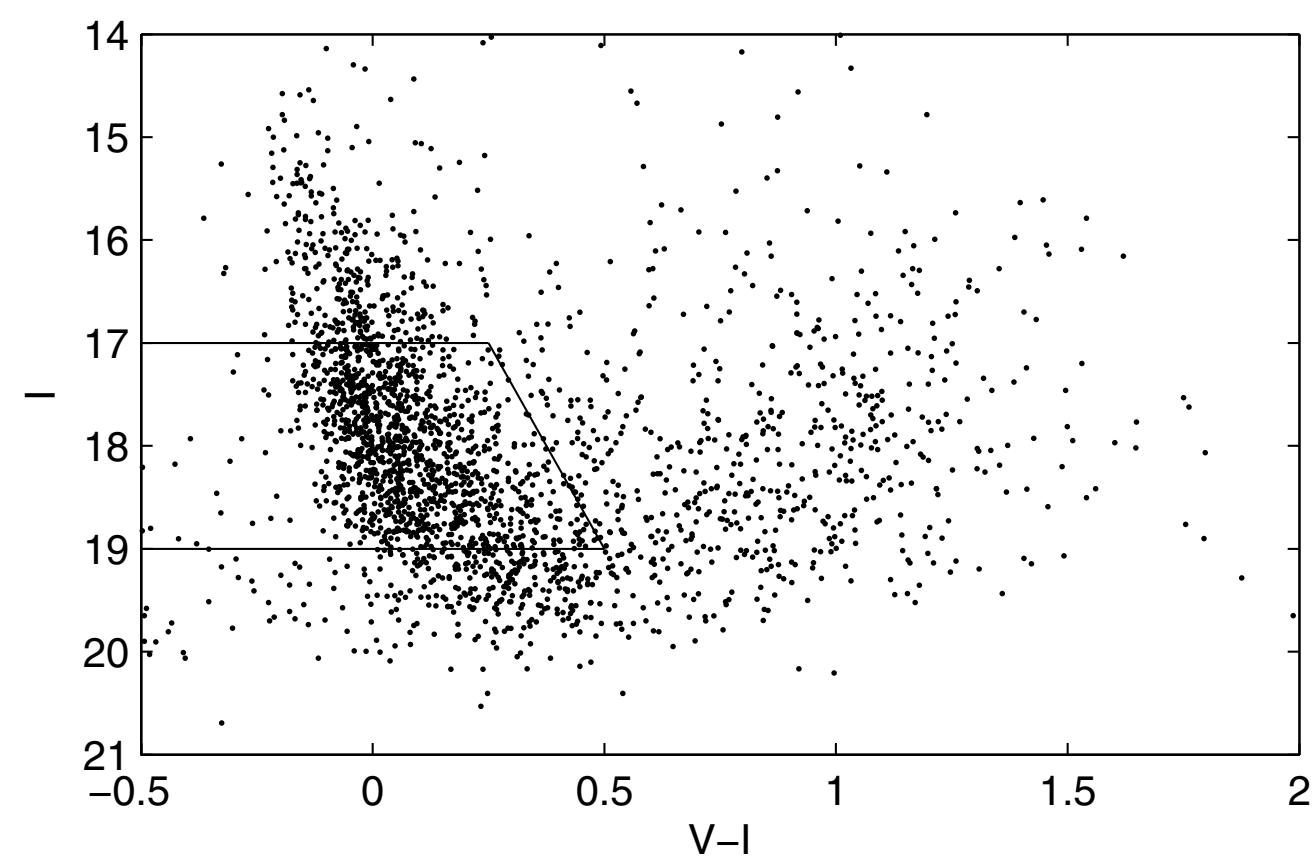

Figure 1. The $I$-magnitude as a function of the averaged $V-I$ colour of all eclipsing binaries of the sample. Only binaries that appear inside the trapezoid were included in the trimmed sample.

Figure 1). In this way we chose only systems with A and B main-sequence primaries. See Mazeh, Tamuz \& North (2006) for a full description of the trimmed sample.

\subsection{The period distribution}

In order to explore the period distribution of the LMC short-period binaries, and not just the eclipsing binaries, we needed to correct for the selection effects which affect the likelihood of a binary system to be eclipsing, and to be detected by the OGLE survey. We did this by calculating for each eclipsing binary in the trimmed sample a weight $w$, which was the reciprocal of the probability of being detected as an eclipsing binary, assuming random orientation and phase (see Tamuz, Mazeh \& North 2006). When calculating the period distribution of the binary population, we then considered that eclipsing binary as $w$ systems.

We plotted in Figure 2 two period histograms of the trimmed sample, before and after the correction for the observational effects was applied. We emphasize that if the correction was applied properly, the right panel represents the period distribution of all binaries in the LMC with $I$ magnitude between 17 and 19, and with sum of radii smaller than 0.6 , and not only the eclipsing binaries.

The corrected period histogram shows clearly a distribution that rises up to about $\log P=0.3$, and then flattens off, suggesting that the period distribution of the shortperiod binaries with early-type binaries is consistent with a flat log distribution between 2 and 10 days.

\subsection{The frequency of the short-period binaries}

As stated above, the corrections applied by Mazeh, Tamuz \& North 2006 allowed us to study the distributions of all short-period binaries, up to 10 days, and not only the 

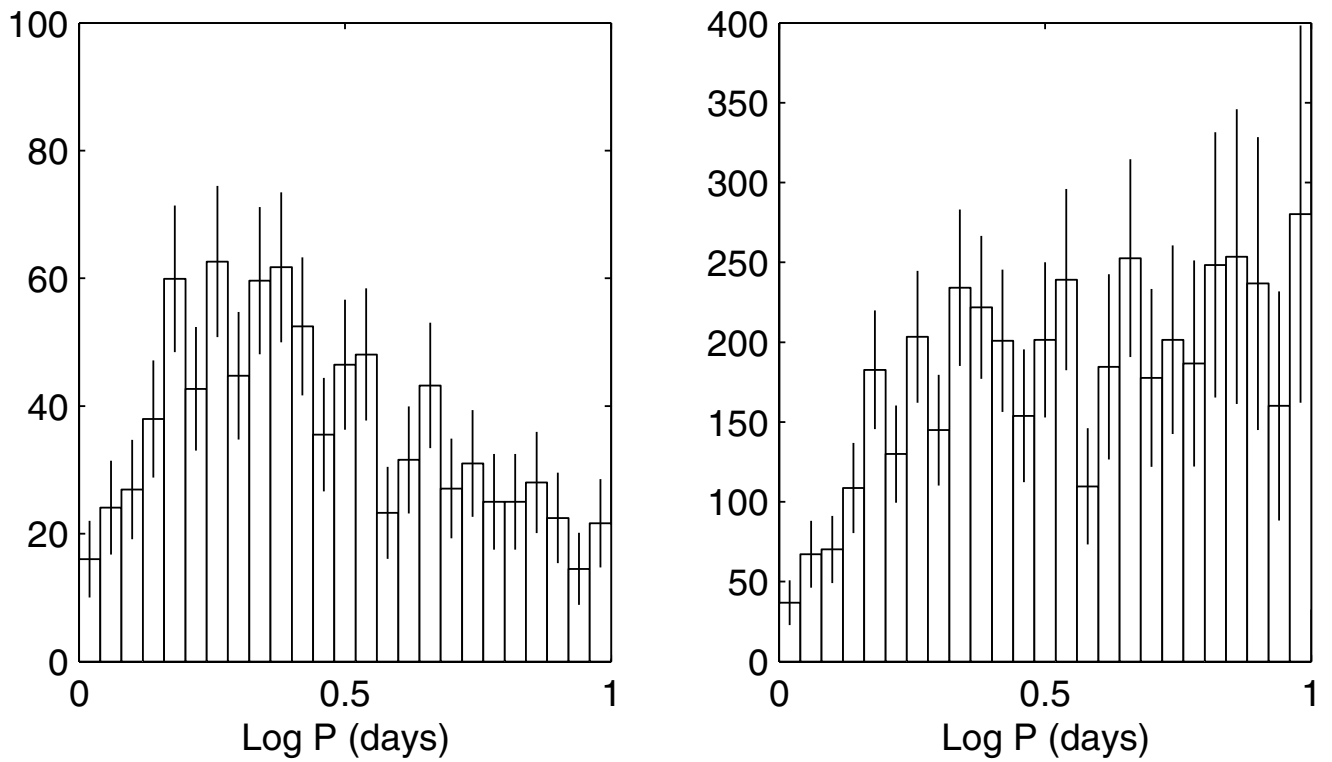

Figure 2. The period distribution of the binaries in the LMC. The left panel shows the period histogram of the trimmed sample, while the right one shows the corrected histogram

eclipsing binaries. We wished to take advantage of this feature of our analysis and estimate, within the limits of the sample, the total number of binaries in the LMC and their fractional frequency.

The sum of weights of the 938 binaries in the trimmed sample was 4585 . This means that to our best estimate there are 4585 short-period binaries in the LMC that fulfill the constraints we had on the trimmed sample. To get the total number of binaries with periods shorter than 10 days we had to add the high-alarm binaries and the ones with large radii; since the added systems are close binaries, their detection probability reaches almost unity so we did not correct for undetected binaries in their case. When we added those, we ended up with 5004 binaries.

We therefore suggest that the number of binaries in the LMC, with period shorter than 10 days, with $I$ between 17 and 19, and for which the $V-I$ colour of the system indicates a main-sequence primary, is about 5000. Assuming Wyrzykowski et al. (2003) might not have detected all the eclipsing binaries in the dataset and classified them as such, we arbitrarily assigned an error of $30 \%$ to the number of binaries in the sample.

In order to estimate the fractional frequency of A- and B-type main-sequence stars which reside in binaries that could have been detected by OGLE, we had to estimate how many main-sequence single stars were found by OGLE in the same range of magnitudes and colours. To do that, we applied exactly the same trimming procedure we performed above to the whole OGLE dataset of LMC stars and found 332,297 main-sequence stars in the $I$-range of 17-19 mag. However, the luminosity of a binary is brighter than the luminosity of its primary by a magnitude that depends on the light ratio of the two stars of the system. Therefore, we considered stellar range of $I=17.5-19.5$, and found 705,535 stars. We therefore adopted 700,000 as a representative number of single stars in the LMC similar to the population of our binary sample. We arbitrarily assign an error of $50 \%$ to this figure.

We therefore concluded that $(0.7 \pm 0.4) \%$ of the main-sequence A- and B-type stars in the LMC are found in binaries with periods shorter than 10 days. 


\section{Discussion}

\subsection{The period distribution}

The flat log-period distribution derived here is probably not consistent with the period distribution of Duquennoy \& Mayor (1991), who adopted a Gaussian with $\overline{\log P}=4.8$ and $\sigma=2.3, P$ being measured in days. Their distribution would provide within the same $\log \mathrm{P}$ interval almost twice as many systems at $\log P=1.0$ than at $\log P=0.3$ (more precisely, the factor would be 1.7), while the new derived distribution is probably flat.

It is interesting to note that Heacox (1998) reanalysed the data of Duquennoy \& Mayor (1991) and claimed that $f(a)$, the distribution of the G-dwarf orbital semi-major axis, $a$, is $f(a) \propto a^{-1} d a$, which implies a flat log orbital separation distribution. This is equivalent to the present probable result, although the latter refers to LMC binaries with A- and B-type primaries, and is limited only to a very small range of orbital separation.

The flat log-period distribution implies a flat log orbital-separation distribution for a given total binary mass. Such a distribution indicates that there is no preferred length scale for the formation of short-period binaries (Heacox 1998), at least in the range between 0.05 and $0.16 \mathrm{AU}$, for a total binary mass of $5 M_{\odot}$. Alternatively, the results indicate that the specific angular momentum distribution is flat on a log scale at the $10^{19}$ $\mathrm{cm}^{2} \mathrm{~s}^{-1}$ range.

\subsection{The binary fraction}

The fractional frequency found here is surprisingly small - $(0.7 \pm 0.4) \%$. It would be interesting to compare the frequency of B-star binaries found here for the LMC with a similar frequency study of B stars in our Galaxy. However, such a large systematic study of eclipsing binaries is not available. Instead, a few radial-velocity and photometric searches for binaries in relatively small Galactic samples were performed. Wolff (1978) studied the frequency of binaries in sharp-lined B7-B9 stars. Her Table 1 presents 73 such stars with $V \sin i<100 \mathrm{~km} \mathrm{~s}^{-1}$, among which 17 are members of binary systems with $P_{\text {orb }}<10$ days. Her sample yields a percentage of $23 \%$, one and a half orders of magnitude larger than what we found in the LMC.

Similar and even higher values of binary frequency were derived for B stars in Galactic clusters and associations. Morrell \& Levato (1991) have determined the frequency of binaries among B stars in the Orion OB1 association, and obtained an overall frequency of binaries with $P_{\mathrm{orb}}<10$ days of $26 \pm 6 \%$. Garcia \& Mermilliod (2001) found a rate of binaries as high as $82 \%$ among stars hotter than B1.5 in the very young open cluster NGC 6231, though in a sample of 34 stars only. Most orbits have periods shorter than 10 days. Raboud (1996) estimated a rate of binaries of at least $52 \%$ for 36 B1-B9 stars in the same cluster.

These studies show that binaries hosting B-type stars tend to be more frequent in young Galactic clusters than in the field. However, even though our LMC binaries are representative of the LMC field rather than of LMC clusters, the frequency we derived appears much lower than that of the binaries in the Galactic field.

A few photometric studies were devoted to the search of eclipsing binaries in Galactic globular clusters (e.g., Yan \& Reid 1996, Kaluzny 1997). One such study was the HST 8.3-day observations of 47 Tuc (Albrow et al. 2001), which monitored 46,422 stars and discovered 5 eclipsing binaries with periods longer than about 4 days. From their conclusion we derive $(2 \pm 1) \%$ for periods between 2 and 10 days. This value, derived for late-type stars, is much smaller than the frequency derived by the radial-velocity surveys of B stars, and is close to the $(0.7 \pm 0.4) \%$ frequency we derived for the LMC. 
We conclude, therefore, that the frequency of binaries we found in the LMC is substantially smaller than the frequency found by Galactic radial-velocity surveys. The detected frequency of B-type binaries is larger by a factor of 30 or more than the frequency we found, while the binary frequency of K- and G-type stars is probably larger by a factor of four (Halbwachs et al. (2003) found $2.7 \pm 0.8$ per cent binaries with $P<10$ days in the solar neighbourhood). On the other hand, the binary frequency found by photometric searches in 47 Tuc is only slightly higher and still consistent with the frequency we deduced for the LMC. It seems that the frequency derived from photometric searches is consistently smaller than the one found by radial-velocity observations. We are not aware of any observational effect that could cause such a large difference between the radial velocity and the photometric studies. Therefore, we suggest that the large difference in the binary frequencies is probably real and remains a mystery. Obviously, it would be extremely useful and interesting to have studies of eclipsing binaries similar to the present one in our Galaxy, as well as in other nearby galaxies.

\section{Acknowledgments}

We are grateful to the OGLE team, and to L. Wyrzykowski in particular, for the photometric data set and for the eclipsing binary analysis. This work was supported by the Israeli Science Foundation through grant no. 03/233.

\section{References}

Albrow, M.D., Gilliland, R.L., Brown, T.M., Edmonds, P.D., Guhathakurta, P., \& Sarajedini, A. $2001, A p J 559,1060$

Duquennoy, A. \& Mayor, M. 1991, A\&A 248, 485

Etzel, P.B. 1981, in Photometric and Spectroscopic Binary Systems, p. 111-120

Garcia, B. \& Mermilliod, J.-C. 2001, A\&A 368, 122

Halbwachs, J.L., Mayor, M., Udry, S., \& Arenou, F. 2003, A\&A 397, 159

Heacox, W.D. 1998, AJ 115, 325

Kaluzny, J., Krzeminski, W., Mazur, B., Wysocka, A., \& Stepien, K. 1997, Acta Astronomica 47,249

Mazeh, T., Tamuz, O., \& North, P. 2006, MNRAS 367, 1531

Morrell, N. \& Levato, H. 1991, ApJS 75, 965

Popper, D.M. \& Etzel, P.B. 1981, AJ 86, 102

Raboud, D. 1996, A\&SA 315, 384

Tamuz, O., Mazeh, T., \& North, P. 2006, MNRAS 367, 1521

Udalski, A., Szymanski, M., Kubiak, M., Pietrzynski, G., Soszynski, I.,Wozniak, P., \& Zebrun K. 2000, Acta Astronomica 50, 307

Wolff, S.C. 1978, ApJ 222, 556

Wyrzykowski, L., Udalski, A., Kubiak, M., Szymanski, M., Zebrun, K., Soszynski, I., Wozniak, P.R., Pietrzynski, G., \& Szewczyk, O. 2003, Acta Astronomica 53, 1

Yan, L. \& Reid, I.N. 1996, MNRAS 279, 751

Zebrun, K., Soszynski, I., Wozniak, P.R., Udalski, A., Kubiak, M., Szymanski, M., Pietrzynski, G., Szewczyk, O., \& Wyrzykowski, L. 2001, Acta Astronomica 51, 317 